\section{Measurement Uncertainty}

Han Haitjema

Mitutoyo RCE, Best, The Netherlands

\section{Synonyms}

Measurement uncertainty; Uncertainty of measurement

\section{Definition}

A nonnegative parameter characterizing the dispersion of the quantity values being attributed to a measurand, based on the information used (Source: JCGM 200:2012 (2012) (VIM)).

\section{Theory and Application}

\section{Introduction}

Few subjects have generated so much debate in the recent decennia as the concept "uncertainty," better specified as "measurement uncertainty." The need for a harmonized approach came from industry, standardization organizations, accreditation organizations, national metrology institutes, etc.

Historically, measurement uncertainty is closely related to the progress of science and adoption/rejection of theories. From the industrial side, there is the fitting of products or, if that cannot be done directly, specifying products and testing whether these specifications are met.

On the other hand, there is the field of statistics, taking samples out of a - limited or unlimited - range of possible outcomes and estimating what the distribution will be behind the outcomes when a limited set - down to one - of outcomes is available.

\section{Basic Documents}

Where in the past - and still - physicists are taught "correct for systematic errors and add all random errors (i.e., standard deviations) quadratically," it was not uncommon that mechanical engineers had as a rule "add all maximum errors to minimize the risk." Nowadays, it is agreed that "systematic" and "statistical" errors are not so essentially different and that a harmonized approach is possible. The approach as outlined in the "GUM" (GUM 1995; JCGM 100:2008 2008) is most generally agreed and available. This document has a supplement 1 (JCGM 101:2008 2008) that looks less basic from the title; but, in fact, this second part is considered the most fundamental basis. The difference is that in supplement 1 , uncertainty is basically treated as the propagation of uncertainty distributions, rather than uncertainties, and that these can be simulated by Monte Carlo methods.

The approach of these documents may look complicated, and for this reason, several handson documents have appeared that claim to have a more practical approach. Typical examples are the 
EA-document EA-4/02 M (2013) and the "PUMA" approach explained in ISO 14253-2 (2011).

\section{Basic Method}

In practice, the precise uncertainty distribution, correlation, and degrees of freedom considerations may not be too relevant, and we will omit these aspects from now on. With the risk of simplifying things too much and without the pretention of giving a definite simplified approach, the basic method can be summarized as follows:

In general, a measurement result $y$ is a function of $n$ input quantities $x_{i}(i=1,2, \ldots n)$. These input quantities can be measured values, known constants, etc. This leads to the general functional relationship, known as the "model function" (EA-4/02 M 2013):

$$
y=f\left(x_{1}, x_{2}, \ldots x_{n}\right)
$$

The model function incorporates the measurement and the calculation procedure. It can be an analytical function, but also a complicated, iterative, computer algorithm. The measurement data $x_{i}$ can be grouped into two categories, depending on the way they, and their uncertainty, are obtained:

(a) Quantities where the value and its uncertainty are directly obtained from the measurements

(b) Quantities where the uncertainties are obtained from other sources, such as calibration data, used material constants, previous measurements.

However, this grouping does not influence the uncertainty evaluation; it is just essential that a standard uncertainty $u_{x i}$ is attributed to any influencing quantity $x_{i}$.

The quantity $Y$ is best approximated by using the measurement result $y$, calculated from best approximations for $\mathrm{X}_{i}: x_{i}$, which are usually the measured data, in Eq. 1.

Now, the uncertainty $u y$ can be written as:

$$
\begin{array}{r}
u_{y}^{2}=\sum_{i}\left(\left[\frac{\partial y}{\partial x_{i}}\right]_{y}\right)^{2} \cdot\left\langle\Delta_{i}^{2}\right\rangle+2 \\
\cdot \sum_{i<j}\left[\frac{\partial^{2} y}{\partial x_{i} \cdot \partial x_{j}}\right] \cdot\left\langle\Delta_{i} \cdot \Delta_{j}\right\rangle
\end{array}
$$

where $\Delta_{i}$ and $\Delta_{j}$ are the deviations from their true value $X_{i}$ of $x_{i}$ and $x_{j}$, respectively; and $<>$ denotes the average over a large ensemble. The squared expected deviation of $x_{i}$ from its real value $<\Delta_{i}^{2}>$ is known as the variance, which is the square of the standard uncertainty $u_{i}$. So $u_{i}^{2}=<\Delta_{i}^{2}>$. The product $\left\langle\Delta_{i} \cdot \Delta_{j}\right\rangle$ is known as the covariance of the deviations in $x_{i}$ and $x_{j}$. In the case of uncorrelated measurement data $x_{i}$ and $x_{j}$, Eq. 2 reduces to:

$$
u_{y}^{2}=\sum_{i}\left(\left[\frac{\partial y}{\partial x_{i}}\right]_{y}\right)^{2} \cdot u_{x_{i}}^{2}
$$

This implies that the expected effects of all influencing factors on the measurement result $y$ are added quadratically. It is common, e.g., in EA-document EA-4/02 M (2013), to set up this calculation in the form of a table as it is shown in Table 1. In the table, we denoted the contributions from the individual influencing factors with $\Delta_{i}$ as these deviations can be both positive and negative. They can also be written as $u_{i}$ with $u_{i}=\left|\Delta_{i}\right|$; where all contributions are squared in the end, this makes no difference.

Such a table is known as an "uncertainty budget." It gives a rapid overview of all influencing factors and their influences. From this, it can easily be seen which factors can best be decreased in order to achieve a lower (better) final uncertainty. For laboratories seeking accreditation, it is in general compulsory that uncertainty budgets are made for every quantity one is accredited for.

\section{Monte Carlo Method}

In the case of many measurement data and complicated measurements, it can be impracticable to set up a full uncertainty budget which includes all quantities. Instead of varying the quantities one by one, as it is shown in Table 1 , one can vary all 
Measurement Uncertainty, Table 1 General form of an uncertainty budget

\begin{tabular}{l|l|l|l|l}
\hline $\begin{array}{l}\text { Quantity } \\
X_{i}\end{array}$ & Value of quantity $x_{i}$ & Standard uncertainty $u\left(x_{i}\right)$ & $\begin{array}{l}\text { Sensitivity } \\
\text { coefficient } c_{i}=\frac{\partial y}{\partial x_{i}}\end{array}$ & $\begin{array}{l}\text { Contribution to standard } \\
\text { uncertainty in } y \\
c_{i} \cdot u\left(x_{i}\right)=\Delta_{i}(y)\end{array}$ \\
\hline$X_{1}$ & $x_{1}$ & $u\left(x_{1}\right)$ & $c_{1}$ & $\Delta_{1}(y)$ \\
\hline$X_{2}$ & $X_{2}$ & $u\left(x_{2}\right)$ & $c_{2}$ & $\Delta_{2}(y)$ \\
\hline$:$ & $:$ & $:$ & $:$ & $:$ \\
\hline$X_{N}$ & $x_{N}$ & $u\left(x_{N}\right)$ & $c_{N}$ & $\Delta_{n}(y)$ \\
\hline$Y$ & $y$ & & Total: & $u(y)=\sqrt{\Delta_{1}^{2}+. .+\Delta_{n}^{2}}$ \\
\hline
\end{tabular}

parameters simultaneously. As the sign of all deviations is not determined, the different influencing factors can both amplify and weaken each other. If we define a random number $r$ as having an average of 0 and a standard deviation of 1 , so $\langle r\rangle=0,\left\langle r_{i} \cdot r_{j}\right\rangle=\delta_{i, j}$ (d being the Kronecker $\delta$-symbol with $\delta_{i j}=1$ for $i=j$, otherwise 0 ) and $\left\langle r^{2}\right\rangle=1$, where $<>$ denotes the average over a large ensemble, then we can simulate a measurement result by varying all input quantities at a time, as following:

$$
\begin{aligned}
y_{r}= & y\left(x_{1}+r_{1} \cdot u\left(x_{1}\right), x_{2}+r_{2} \cdot u\left(x_{2}\right), \ldots ., x_{N}\right. \\
& \left.+r_{N} \cdot u\left(x_{N}\right)\right)
\end{aligned}
$$

The average of $y_{r}$ is the expected value of $y$, and the standard deviation of $y_{r}$ is the standard deviation of $y$ that can be used as a measure of the uncertainty. Proofs of this are given by Haitjema (2011).

The number of simulations $(K)$ needed depends on the purpose of the simulations. If one wants to simulate different distributions for each quantity, and consider the final uncertainty distribution, then some hundreds of simulations are needed. For a Gaussian distribution, an estimate for the "standard deviation in the standard uncertainty" $s(u)$ is given by:

$$
s(u)=\frac{u}{\sqrt{2 K-2}}
$$

This means that if one aims to have a "simulation" uncertainty of about $10 \%$ of the estimated uncertainty, some 50 simulations must be carried out
$(K=50)$. Herewith, it must taken into consideration that out of their nature, the uncertainties which act as input for the simulations are seldom estimated much better than $30 \%$.

This method of calculation of uncertainties has the following characteristics and possibilities:

- The uncertainty distribution can be determined where different input quantities have different distributions

- One can choose either to determine the standard uncertainty or to calculate the $95 \%$ confidence interval. In the latter case, one must consider the distribution of some hundreds of simulations.

- The method accounts for higher-order terms if the system formula contains products of terms with nominal values of 0 .

- The reality can well be approximated by keeping an "unknown systematic error" constant in each simulation, but vary it between simulations. Examples are probe diameters, temperatures and temperature gradients, calibrationuncertainties of material measures, etc.

- A known systematic error which is not corrected can be simulated with its value taken as the standard uncertainty and the random number $r= \pm 1$. This can be considered as a bi-modal distribution (Tyler Estler 1999).

- Correlations between quantities can be simulated by correlating the used random numbers.

These characteristics make the Monte Carlo simulation method, a potential tool for estimations of uncertainties which are not possible when using 
the mainstream GUM uncertainty budget. Especially for complicated measurements, such as carried out by a CMM, or by form measuring instruments, it is about the only feasible method. Some earlier publications on this subject in dimensional metrology were given by Schwenke (1999; Schwenke et al. 2000). More specifically about CMMs, a European research project was carried out (Trapet et al. 1999). As a follow-up of this, several participants continued the research (Balsamo et al. 1999; van Dorp et al. 2001). This research was summarized by Wilhelm et al. (2001). The general concept of using Monte Carlo method in uncertainty evaluation is described by Cox et al. (2001).

\section{Uncertainty and Confidence Intervals}

Uncertainty can be expressed as a standard uncertainty $u(y)$. The standard uncertainty can be multiplied by a number $k$ such that it gives a confidence interval for the measurand $Y$. Then the probability of $Y$ to be in the region $[y-k \cdot u(y) ; y+k \cdot u(y)]$ can be expressed as a percentage, and the region is called the confidence interval. With some approximations and the assumption of a Gaussian distribution, it can be stated that taking $k=2$ will generate a confidence interval of approximately $95 \%$.

It can be discussed what is most useful: just the standard uncertainty or a confidence interval. As an end result, the confidence interval is most useful for the user; however, if the uncertainty is to be used in other uncertainty calculations (i.e., $y$ becomes one of the quantities $x$ in a further measurement process), the standard uncertainty is more useful.

\section{Use of Uncertainty}

It is generally stated that a measurement result without indication of uncertainty has no value. However, how and for what this uncertainty is useful or can be used may vary. We give a few possibilities here:

\section{Testing of Scientific Theories}

In order to prove that a theory must be wrong or to demonstrate the superiority of the one theory against the other, it is the uncertainty in the measurement that enables a judgment. For a recent example, an experiment can give as a result that neutrinos travel faster than the speed of light, but the real scientific debate starts if their speed is faster than the speed of light when the measurement uncertainty is taken into account with a significant confidence interval.

\section{Testing Against Specifications}

If the geometry of a product must satisfy certain specifications, it must do so including its uncertainty interval. If the uncertainty interval overlaps the specification limit, there is a problem. The probability of this problem can be reduced by reducing the uncertainty, but it cannot be eliminated. In ISO 14253-1 (2013), it is stated that the uncertainty is always a disadvantage for the interested party. So a manufacturer wanting to sell his instrument must show that this instrument can meet the specification including the measurement uncertainty interval. For a customer who wants to complain that a machine is outside its specification, the measurement result including its confidence interval must be outside the specification limits.

\section{Calibration of Standards and Measurement Instruments}

The two actions mentioned previously can only be carried out using calibrated instruments and standards. The uncertainty can only be calculated if the uncertainty of these calibrations is known.

\section{Uncertainty and Traceability}

Uncertainty and traceability appear to be different concepts, but, in fact, they are identical twins. If a measurement is traceable, an uncertainty budget can be made that includes the calibration of the reference standard. The uncertainty of this reference standard can only be properly stated if it is traceable to a primary standard. On the other hand, if no uncertainty calculation can be made, a measurement cannot be traceable, and if a measurement is not traceable, no uncertainty can be calculated as the uncertainty of the used references is not known. 


\section{Cross-References}

$\checkmark$ Accuracy

- Calibration

$\checkmark$ Error

- Traceability

\section{References}

Balsamo A, Di Ciommo M, Mugno R, Rebaglia BI, Ricci E, Grella R (1999) Evaluation of CMM uncertainty through Monte Carlo simulations. Ann CIRP Manuf Technol 48(1):425-428

Cox MG, Dainton MP, Harris PM (2001) Software specifications for uncertainty calculation and associated statistical analysis. National Physics Laboratory (NPL) report CMSC 10/01. National Physics Laboratory, Teddington

EA-4/02 M (2013) Expression of the uncertainty of measurement in calibration, European co-operation for accreditation. http://www.european-accreditation.org/ publication/ea-4-02-m-rev01-september-2013. Accessed 14 March 2016

GUM (1995) Guide to the expression of uncertainty in measurements. ISO, Geneva

Haitjema H (2011) Task specific uncertainty estimation in dimensional metrology. Int J Precis Technol 2:226-245

ISO 14253-1 (2013) Geometrical product specifications (GPS) - inspection by measurement of workpieces and measuring equipment, part 1: decision rules for proving conformance or non-conformance with specifications. ISO, Geneva

ISO 14253-2 (2011) Geometrical product specifications (GPS) - inspection by measurement of workpieces and measuring equipment. Part 2: guidance for the estimation of uncertainty in GPS measurement, in calibration of measurement equipment and in product verification. ISO, Geneva, 1998

JCGM 100:2008 (2008) Evaluation of measurement data - guide to the expression of uncertainty in measurement (GUM 1995 with minor corrections). Bureau International des Poids et Mesures
JCGM 101:2008 (2008) Evaluation of measurement data - supplement 1 to the 'Guide to the expression of uncertainty in measurement' - propagation of distributions using a Monte Carlo method. Bureau International des Poids et Mesures. http://www.bipm.org/utils/com mon/documents/jcgm/JCGM_101_2008_E.pdf. Accessed 14 March 2016

JCGM 200:2012 (2012) International vocabulary of metrology: basic and general concepts and associated terms (VIM). Bureau International des Poids et Mesures. http://www.bipm.org/utils/common/docume nts/jcgm/JCGM_200_2012.pdf. Accessed Mach 14 2016; Recent update: JCGM:200:2012

Schwenke H (1999) Abschätzung von messunsicherheiten durch simulation an beispielen aus der fertigungsmesstechnik [A simulation-based approach for the estimation of measurement uncertainties in the area of prodcution measurement technology], Thesis, PTB-F36, Physikalisch-Technische Bundesanstalt, Braunschweig (in German)

Schwenke H, Siebert BRL, Wäldele F, Kunzmann $H$ (2000) Assessment of uncertainties in dimensional metrology by Monte Carlo simulation, proposal of a modular and visual software. CIRP Ann 49(1):395-398

Trapet E, Franke M, Härtig F et al (1999) Development of methods and guidelines to establish traceability for measurements with coordinate measuring machines, EU project contract no. MAT1-CT94.0076, European Commision bcr information project report Applied Metrology, Directorate-General Science, Research and Development

Tyler Estler W (1999) Measurement as inference: fundamental ideas. CIRP Ann 48(2):611-631, keynote paper van Dorp B, Delbressine FLM, Haitjema H, Schellekens PHJ (2001) Calculation of measurement uncertainty for multi-dimensional machines, using the method of surrogate data. In: Ciarlini $\mathrm{P}$ et al (eds) Advanced mathematical and computational tools in metrology V. World Scientific Publishing, Singapore, pp 344-351

Wilhelm RG, Hocken R, Schwenke H (2001) Task specific uncertainty in coordinate measurement. Ann CIRP Manuf Technol 50(2):553-563 